\title{
A Systematic Review on the Impact of Genetic Testing for Familial Melanoma I: Primary and Secondary Preventative Behaviours
}

\author{
Clare A. Primiero ${ }^{a}$ Tatiane Yanes $^{a} \quad$ Anna Finnane $^{b} \quad$ H. Peter Soyer ${ }^{a}$ c \\ Aideen M. McInerney-Leo ${ }^{\mathrm{a}}$ \\ ${ }^{a}$ The University of Queensland Diamantina Institute, The University of Queensland, Dermatology Research

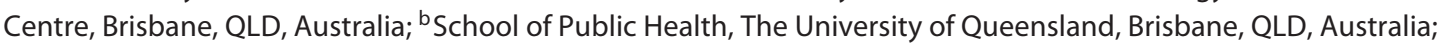 \\ 'Department of Dermatology, Princess Alexandra Hospital, Brisbane, QLD, Australia
}

\section{Keywords}

Melanoma - Genetic testing · Preventative behaviour .

Surveillance

\begin{abstract}
Background: Increasing availability of panel testing for known high-penetrance familial melanoma genes has made it possible to improve risk awareness in those at greatest risk. Prior to wider implementation, the role of genetic testing in preventing melanoma, through influencing primary and secondary preventative behaviours, requires clarification. Methods: Database searches of PubMed, Embase, CINAHL, PsycINFO and the Cochrane Library were conducted for studies describing preventative behaviour outcomes in response to genetic testing for melanoma risk. Publications describing original research of any study type were screened for eligibility. Results: Eighteen publications describing 11 unique studies were reviewed. Outcomes assessed are based on health behaviour recommendations for those at increased risk: adherence to sun-protective behaviour (SPB); clinical skin examinations (CSE); skin self-examinations (SSE); and family discussion of risk. Overall, modest increases in adherence to primary prevention strategies of SPB were observed following genetic testing. Importantly, there were no net decreases in SPB found amongst non-carriers. For sec-
\end{abstract}

ondary preventative behaviour outcomes, including CSE and SSE, increases in post-test intentions and long-term adherence were reported across several subgroups in approximately half of the studies. While this increase reached significance in mutation carriers in some studies, one study reported a significant decline in annual CSE adherence of non-mutation carriers. Conclusions: Evidence reviewed suggests that genetic testing has a modestly positive impact on preventative behaviour in high-risk individuals. Furthermore, improvements are observed regardless of mutation carrier status, although greater adherence is found in carriers. While additional studies of more diverse cohorts would be needed to inform clinical recommendations, the findings are encouraging and suggest that genetic testing for melanoma has a positive impact on preventative behaviours.

(c) 2021 S. Karger AG, Basel

\section{Introduction}

The average lifetime risk of cutaneous melanoma is approximately $3 \%$, varying depending on geographical location [1]. However, individuals with mutations in high-penetrance genes, such as CDKN2A, incur a lifetime risk of $52 \%$ [2]. The current capabilities of genetic testing for familial melanoma are still limited, as known

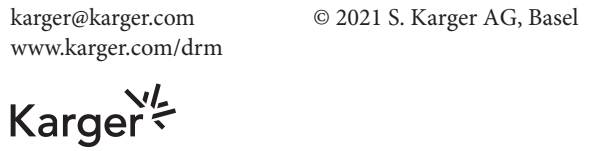

Aideen McInerney-Leo and H. Peter Soyer 


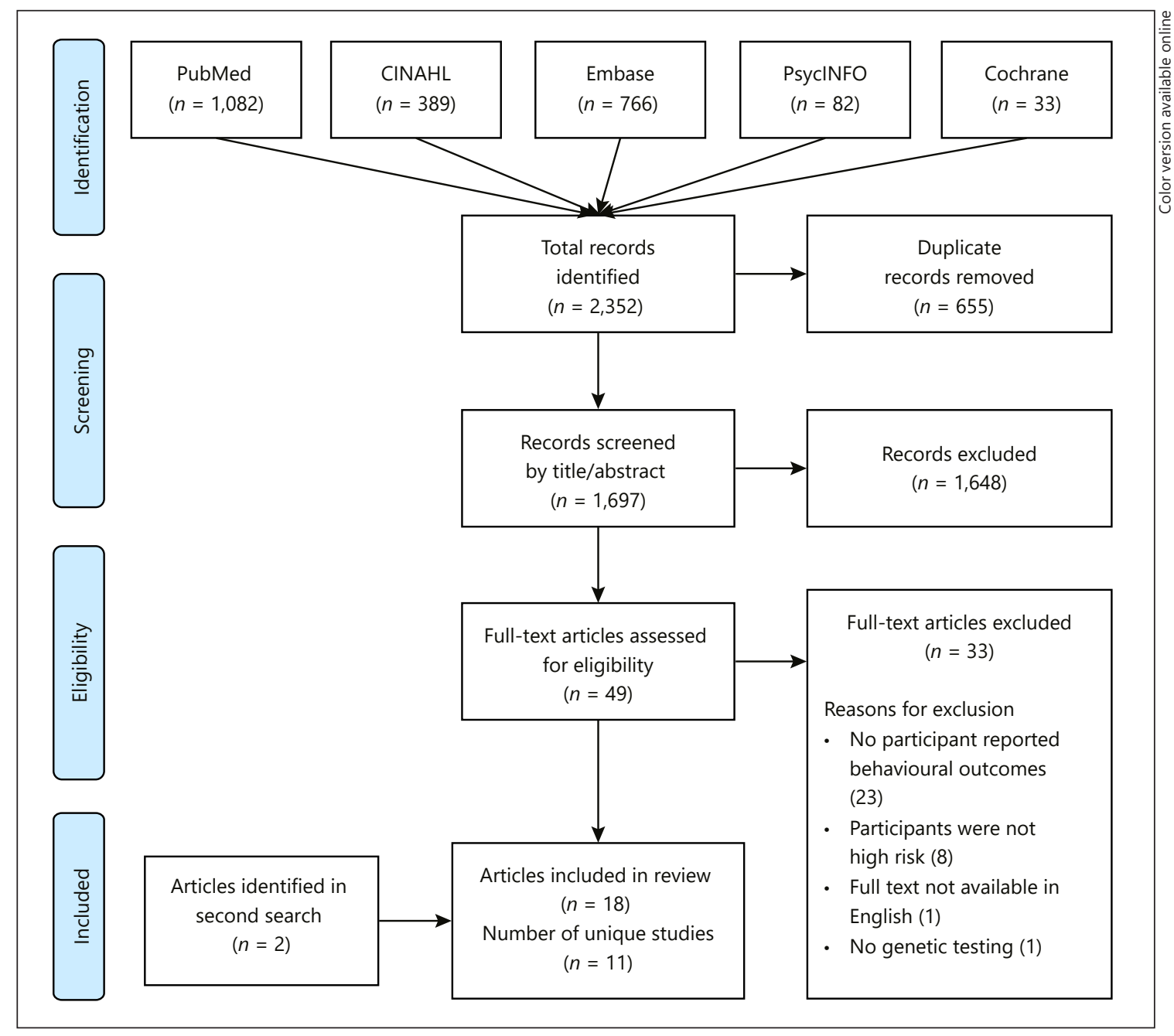

Fig. 1. PRISMA flow chart describing the study selection process.

high-penetrance variants account for approximately $22 \%$ of familial cases, while a causative mutation remains unidentified in the majority of families with a strong history of melanoma [3]. A key objective of providing genetic risk assessment in a familial cancer setting is to reduce disease morbidity and mortality through the uptake of preventative health behaviours and screening interventions [4]. Previous reviews of high-risk individuals have reported that current primary and secondary preventative behaviour is suboptimal [5-8]. Individuals carrying mutations in a high-penetrance gene, such as $C D K N 2 A$, are advised to uphold stringent sun protection through the use of sunscreen, protective clothing and avoiding high UVR exposure (i.e., primary prevention), and to increase chances of early detection through secondary preventative methods including regular clinical skin examinations (CSEs) and skin self-examinations (SSEs) [9].

Consumer interest and expectations are increasing, regarding the potential to incorporate genetic testing to personalise clinical care $[10,11]$. Rapid advancements in genomic technology have seen increasing reliability and affordability in genetic risk analysis for several diseases [12]. However, whether the communication of genetic risk assessment translates to improved health outcomes requires further evaluation [13]. Furthermore, the implementation of genetic services to meet consumer demand raises logistical obstacles that require attention [14]. This is the first of a 2-part series of systematic reviews evaluating outcomes of genetic testing for familial melanoma. The second part assesses the psychosocial impact of testing in this cohort (see part II, DOI: 10.1159/000513576). 
Table 1. Glossary of commonly used terms

\begin{tabular}{ll}
\hline Carrier/non-carrier & $\begin{array}{l}\text { Whether a person has tested positive or negative } \\
\text { for a pathogenic variant (in CDKN2A) }\end{array}$ \\
\hline Affected/unaffected & $\begin{array}{l}\text { Whether a participant has a personal history (of } \\
\text { melanoma) }\end{array}$ \\
\hline Controls & $\begin{array}{l}\text { Participants who received counselling without } \\
\text { genetic testing }\end{array}$ \\
\hline
\end{tabular}

\section{Methods}

This systematic review was conducted according to the Preferred Reporting Items for Systematic Reviews and Meta-Analyses (PRISMA) guidelines [15]. A protocol was registered with PROSPERO (International Prospective Register of Systematic Reviews) and can be accessed at https://www.crd.york.ac.uk/prospero/display_record.asp?ID=CRD42020185688.

A database search using PubMed, Embase, CINAHL, PsycINFO and the Cochrane Library was conducted using search terms related to genetic testing, health behaviour and melanoma risk. Search strings are provided in online supplementary Table S1 (see www.karger.com/doi/10.1159/000513919 for all online suppl. material). The date range January 1995 to June 2020 was applied, to coincide with the discovery of the CDKN2A risk gene. No restrictions on study types were used; however, only original research published in English was assessed. Eligibility criteria included: (i) participants were described as having a high risk of melanoma, (ii) genetic testing (or hypothetical scenarios) for CDKN2A was offered, and (iii) preventative behaviour outcomes were available. Records were screened by reviewing titles/abstracts, and remaining articles were assessed for eligibility by reviewing full texts. A second author performed screening on $10 \%$ of articles. A standardised report form was used for data extraction, including: citation, study design, participant characteristics, outcomes and follow-up time points. Risk of bias and quality of individual studies was assessed using the QualSyst tool by author C.P., with second author T.Y. assessing 25\% of articles [16]. A narrative synthesis of results is presented, as the heterogeneity of study designs and outcomes precluded the quantitative pooling of results [17]. A glossary of common terms used throughout this review is provided in Table 1.

\section{Results}

The database search identified 2,352 records, and after eliminating duplicates, 1,697 remained (Fig. 1). A further 1,590 records were removed after screening titles/abstracts. 49 full-text articles were assessed for eligibility, and 33 were excluded (reasons described in Fig. 1). A second literature search using backwards and forwards citations revealed 2 additional articles $[18,19]$. A total of 18 articles, describing 11 unique studies were included in the final review. Genetic testing for $C D K N 2 A$ risk variants was performed in all studies, except for 2 , which used hypothetical testing scenarios. Study characteristics are described in Table 2. Interrater reliability between authors for the screening and quality assessment produced a kappa score of 0.88 and 0.96 , respectively. A kappa score $>0.8$ is associated with high interrater reliability [20]. Quality scores using the QualSyst tool ranged from 68 to $100 \%$ (online suppl. Table S2). No studies were excluded following quality assessment.

\section{Discussion of Test Results}

Six studies included participant-reported outcomes related to the discussion of genetic test results with family, friends and health care professionals $[18,21-$ $25]$. Discussions occurred primarily with family members, and commonly included risk awareness and prevention strategies. Two studies, which used hypothetical testing scenarios, found that intentions to discuss results with relatives to raise risk awareness were significantly greater in high-risk scenarios and amongst women $[18,21]$. A small American study surveyed participants 3 months after test disclosure and reported that $18 / 19$ had discussed their results with others, including spouse/family (18/19), friends (8/19) and health care providers (6/19) [22]. A larger, more recent study compared participants' intentions to discuss results immediately after disclosure to discussions reported 6 months after disclosure [23]. The study found that $100 \%$ of carriers and $83 \%$ of non-carriers intended to discuss genetic test results with health care providers, and by 6 months $79 \%$ of carriers had, compared to $41 \%$ of non-carriers [23]. However, $100 \%$ of participants had shared their results with at least 1 family member in that time [23]. A Utah study, which recorded participants' discussion of primary prevention with children and grandchildren, found a decline in discussion 6 months after disclosure compared to the pretesting period, especially amongst non-carriers [24]. The most frequently discussed topics included sunscreen use and risky behaviours such as intentional tanning and sun exposure [24]. Participants also reported that resources to aid discussion with children would have been helpful [24]. Lastly, a study providing genetic testing for minors found that, regardless of carrier status, discussion about melanoma prevention increased from the 1-month to 1-year time point for both parents (36-73\%) and children (61-67\%) [25]. 


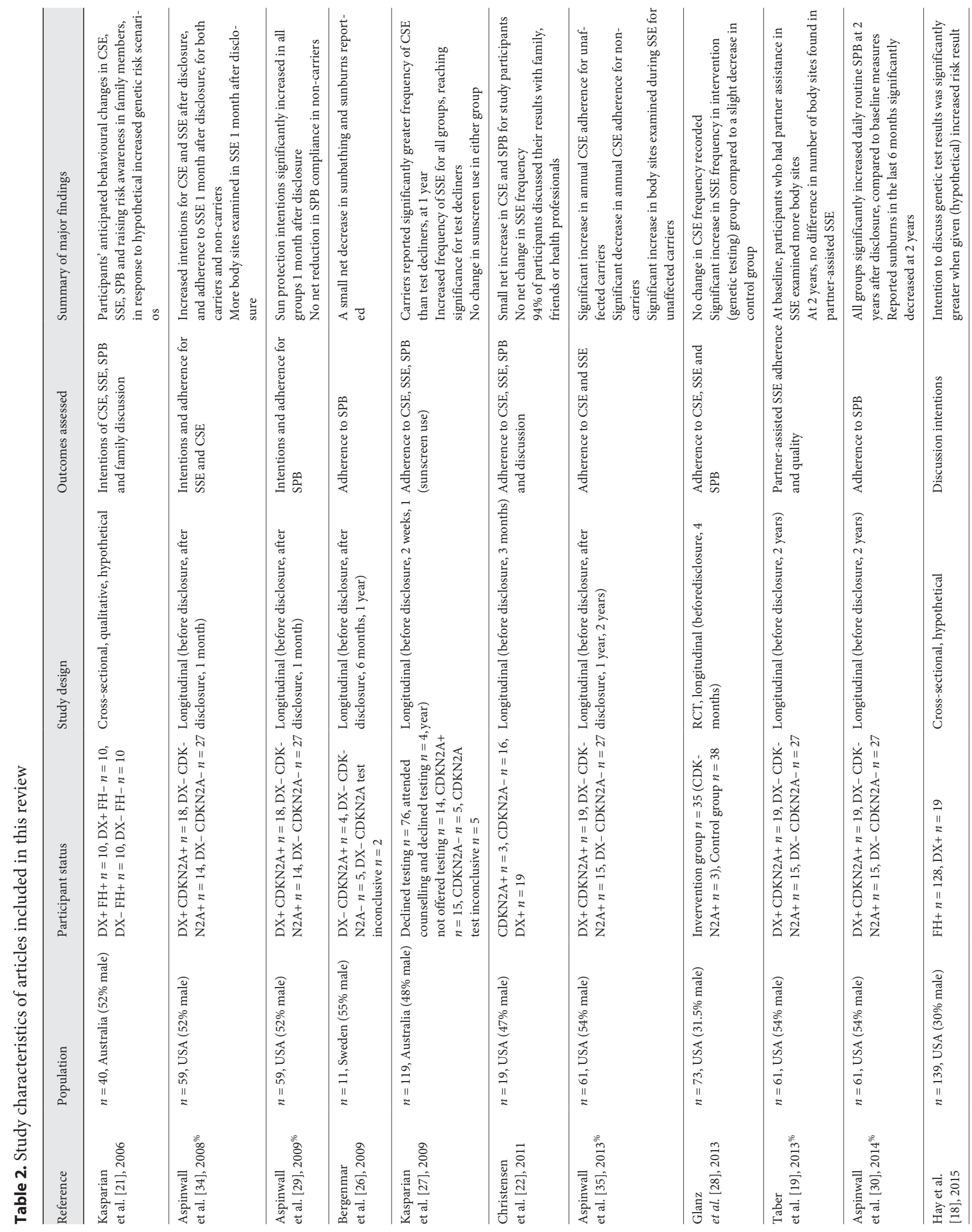




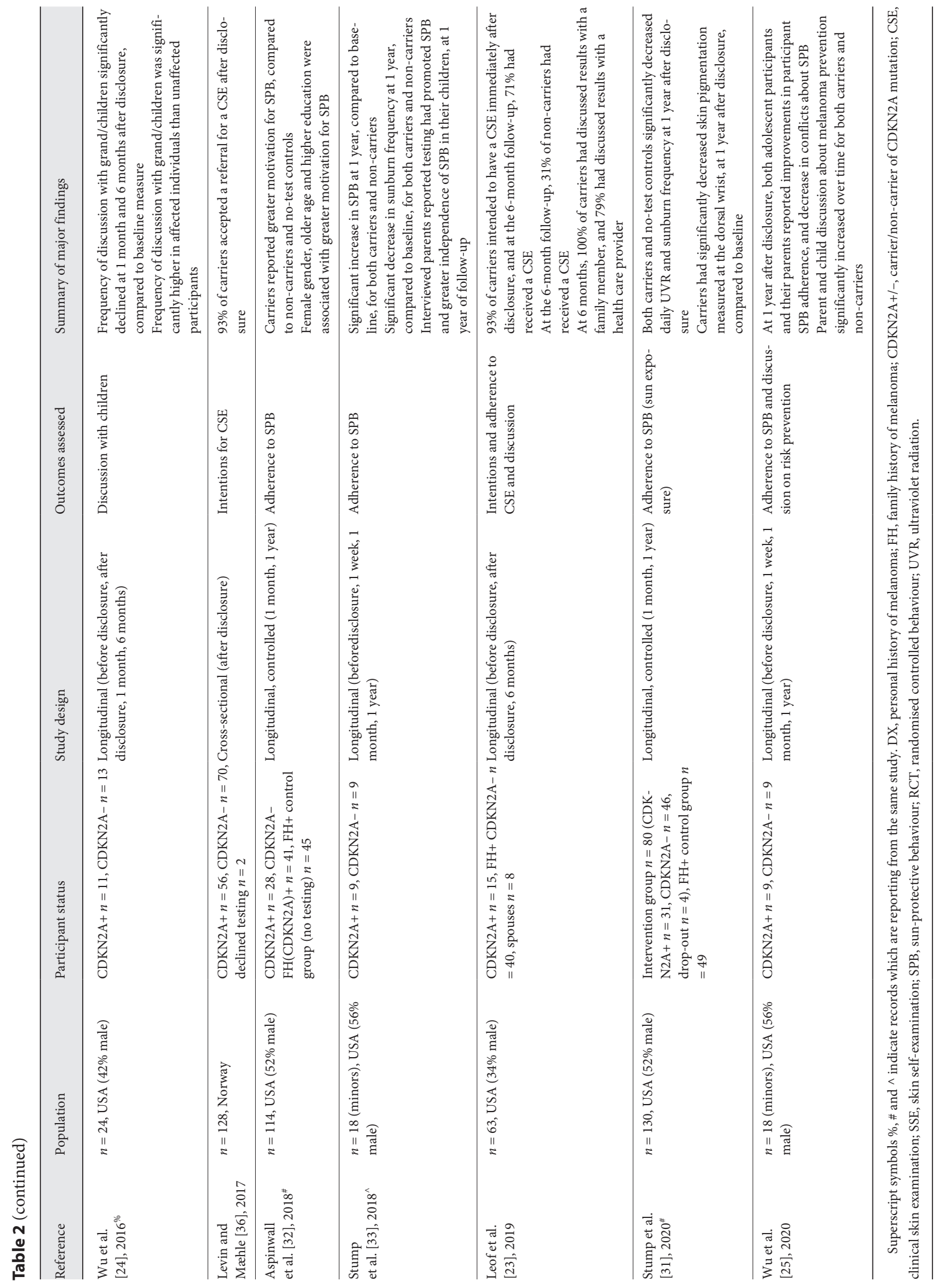




\section{Sun-Protective Behaviour}

Sun-protective behaviour (SPB) was reported in 11 articles, from 8 unique studies [21, 22, 25-33]. Studies assessed a range of SPB, including sunscreen use, protective clothing, avoiding sun exposure and tanning. An early qualitative study, which reported responses to hypothetical genetic testing, found the majority of participants anticipated increasing SPB if found to be mutation carriers [21]. Studies conducted across multiple geographic locations (Sweden [26], Australia [27], USA [22]) reported little or no changes in SPB including sunscreen use [27], tanning [26] and protective clothing [22]. A larger randomised controlled trial $(n=73)$ which measured reported SPB at baseline and 4 months after disclosure found the only statistically significant SPB change was a moderate decrease in "wearing a shirt" amongst the intervention (genetic testing) group compared to controls (no testing) [28]. Due to a limited number of mutation carriers $(n=$ 3 ), outcomes were not evaluated based on carrier status [28]. A study in Utah, USA, statistically analysed participant outcomes based on CDKN2A carrier status and personal history of melanoma (e.g., affected vs. unaffected). The population of this study included $n=18$ affected carriers, $n=14$ unaffected carriers and $n=27$ unaffected non-carriers. At 1 month after disclosure, no overall decline of any SPBs was identified in either group, and 33\% of participants reported incorporating a new SPB into their daily routine [29]. There was a significant increase in daily routine SPB (sunscreen use, UV avoidance and protective clothing), with $97 \%$ of all participants reporting at least one daily SPB behaviour 2 years after disclosure, compared to $78 \%$ at baseline [30]. Two years after disclosure, a significant decrease in reported sunburns for the previous 6 months was identified among unaffected carriers (2.01 at baseline, to 0.60 at 2 years) [30]. A more recent study from Utah followed carriers $(n=31)$, noncarriers $(n=46)$ and "no-test" controls $(n=49)$ for 1 year, using objective measures of UVR meters, melanin index scores measured by reflectance spectrometry, and reported sunburns as an indicator of excessive sun exposure [31]. The study found no difference in facial melanin index scores between groups at follow-up; however, dorsal wrist melanin index scores decreased significantly from baseline in carriers 1 year after disclosure [31]. In addition, both carriers and controls significantly decreased their daily UVR exposure over time, carriers reported fewer sunburns, while no significant change was measured for non-mutation carriers [31]. In this cohort, carriers reported higher SPB motivation than both non-carriers and controls (counselling only) [32]. A third study from Utah recruited 18 minors from CDKN2A-positive families for genetic testing, and reported significant increases in SPB adherence, and a decrease in sunburn frequency in all participants at 1 year [33]. In interviews, $73 \%$ of parents, and $50 \%$ of adolescent participants reported making positive changes to SPB in their family [25]. The majority of mothers agreed that testing had promoted SPB for their children (82\%) and resulted in increased independent practice of SPB (46\%) by their children [33].

\section{SSE Adherence}

Individuals at increased risk for melanoma are recommended to perform monthly SSE. Seven publications reported outcomes for SSE adherence or intentions [19, 21, $22,27,28,34,35], 3$ of which reported results from the same study in Utah, USA $[19,34,35]$. An early qualitative study using hypothetical scenarios reported that most participants intended to increase SSE if identified as a carrier [21]. Three individual studies, which reported longitudinal SSE outcomes for 3 [22], 4 [28] and 12 [27] months after result disclosure reported a trend in increased SSE amongst all participant groups (carriers, non-carriers, no-test controls and test decliners). However, the only statistically significant increase in SSE was measured in the test decliner group, in the 12-month study [27]. A study in Utah reported that carriers' intentions to perform SSE significantly increased from pretesting adherence, and a significant increase in SSE was reported at the 1 -month and 2-year time points [34, 35]. Furthermore, adherence to $\geq 1$ SSE a month increased for unaffected carriers from $30 \%$ before testing to $60 \% 2$ years after disclosure [35]. Similarly, SSE quality increased, with more body sites examined across all groups $[19,35]$. At baseline, partner assistance in SSE was associated with examination of more body sites; however, 2 years after disclosure, no difference was found [19]. Lastly, the Utah study reported participants perceived barriers to performing SSE, including "hard to remember" and "time consuming" [35].

\section{Clinical Skin Examinations}

A total of 8 articles, describing 7 unique studies, reported participant outcomes for intentions and/or adherence to recommended frequency of CSEs [21-23, 27, 28, 34-36]. In a qualitative study evaluating responses to hypothetical scenarios, the majority of participants anticipated increasing CSE adherence if they tested positive [21]. Three studies reported on intentions to have a CSE immediately after receiving genetic test results $[23,34$, 
36]. In one study, $93 \%$ of carriers $(n=52 / 56)$ accepted a referral for an annual CSE [36]. A later longitudinal study reported $93 \%$ of carriers $(n=14 / 15)$ intended to seek a CSE within 6 months [23]. When followed up at 6 months after disclosure, $71 \%$ of carriers and $31 \%$ of non-carriers had received a CSE [23]. A study from Utah reported that intentions for annual CSE increased significantly from current practices amongst all groups immediately after disclosure [34]. At 2 years after disclosure, adherence to an annual CSE had increased significantly for unaffected carriers (from 40 to $70 \%$ ), while unaffected non-carriers showed a significant decrease (from 56 to 13\%) [35]. An Australian study, which compared outcomes of carriers $(n=15)$, non-carriers $(n=5)$ and decliners $(n=76)$ of testing for 1 year, found an increase in CSE frequency in all groups, reporting a significant difference between carriers (85\% adherence) and test decliners (50\% adherence) [27]. Lastly, 2 longitudinal studies from the USA, which followed participants 4 and 3 months after disclosure, reported no significant difference in CSE adherence following testing; however, both studies reported only 3 CDKN2A carriers [22, 28].

\section{Discussion}

The research examining primary prevention outcomes in this review has revealed mixed findings regarding whether the genetic testing process may lead to increased sun-protective behaviour. Three separate studies, all within the state of Utah, USA, reported significant improvements in SPB amongst carriers [30-32]. Similarly, in a study involving minors, non-carriers also showed significant improvements [33]. Importantly, there was no overall decline of SPB reported amongst non-carriers in any study included in the review. Evidence for improved SPB included participant-reported outcomes on sunburn frequency and sun protection, as well as objective measures using UVR meters and skin pigmentation recordings. Although 4 longitudinal studies in this review did not report significant changes in primary prevention after test disclosure, limitations on follow-up period, study sample size or methods used to measure SPB may have contributed to the lack of significant findings [22, 26-28]. It is also useful to acknowledge that those with a previous history tend to have a higher baseline level of SPB, and therefore limited capacity for showing improvements.

When analysing the impact on primary prevention, this review has considered outcomes of participant-reported discussion of test results with others. In public health models, family-focused interventions for promotion of health and disease prevention are becoming increasingly recognised as a strategy towards greater, sustained health outcomes $[37,38]$. In the context of familial melanoma, the potential for a wider impact on primary prevention in relatives of high-risk individuals is particularly relevant for children of mutation carriers. While melanoma is primarily diagnosed in those aged over 40 years, previous research has found that intermittent sun exposure and sunburns in adolescence incur an increased risk of melanoma onset in adulthood [39]. Although it is difficult to measure the effect, the discussion amongst family members following genetic testing is a valuable tool in raising risk awareness and promoting preventative measures within melanoma-prone families. With this considered, it was positive to find discussion with family members under risk and prevention after testing were highly prevalent among study participants.

The current secondary prevention recommendations for high-risk individuals focus on early detection and include monthly SSE and, depending on geographical location and other risk factors, between 3 and 12 monthly CSEs [9]. Previous epidemiological studies have found that non-carriers in families with a confirmed mutation are still at twice the risk of the general population level, and therefore are recommended to maintain annual CSEs $[40,41]$. An increase in CSE intentions and/or adherence amongst carriers was evident in most studies reviewed $[21,23,34-36]$. Two studies that did not report significant differences in CSE adherence had limited follow-up periods of 3 and 4 months afterdisclosure $[22,28]$. Of concern, one study reported a significant decrease in annual CSE in non-carriers [35]. This highlights a challenge in communicating test results, to ensure non-carriers are not left with a false sense of security. This is particularly important, considering that less than a quarter of familial melanoma cases can be attributed to a known gene mutation [3].

There have been mixed results and a lack of consensus regarding clinical utility for genetic testing in several complex diseases. In the breast cancer setting, testing of the $B R C A 1$ and $B R C A 2$ genes is associated with improved survival through the uptake of risk management strategies, such as increased screening and risk-reducing surgery $[42,43]$. One systematic review for various diseases including familial cancers reported an increase in screening adherence in carriers [44], while a recent meta-analysis showed a significant increase in self-reported preventative behaviour change amongst carriers [45]. In contrast, limited uptake of health behaviours such as smoking 
cessation, diet and physical activity has been identified after genetic testing for common complex disorders (e.g., coronary heart disease, Alzheimer disease) [44, 46-49]. Combined, the reviews indicate clinical utility of genetic testing in secondary prevention efforts, by motivating screening adherence and protective clinical decisions; however, evidence for risk-reducing primary prevention is limited. One unanswered question is whether change in sun-protective behaviour is more achievable than other health behaviours such as smoking, diet and exercise where addiction may hinder behaviour change. Furthermore, the direct link between sun exposure and skin cancer is well understood and accepted by most people, when compared to the complex relationships between other health behaviours and disease risk.

Although the evidence of clinical utility of genetic testing in familial melanoma is not strong, the increasing consumer demand is evident [10]. Currently, predictive genetic testing is primarily provided by qualified genetic counsellors, and tests are accompanied by pre- and postgenetic education and counselling. As new genetic discoveries are made, the increasing demand on genetic services is predicted to cause a shortage of qualified workforce [14]. A recent transition to mainstream testing services in specialist clinics, for example, BRCA1 and 2 testing in oncology clinics [50], is one strategy used to address the increased demand [51]. Melanoma genetic testing is an ideal inaugural genetic test to mainstream into dermatology clinics. Benefits include opportunistic targeted screening and education on primary prevention, with the ability to review adherence in the long term at follow-up CSE appointments. Furthermore, a pre-existing patient-doctor relationship may have a positive effect on compliance to risk management recommendations [52]. The future development of a standardised approach for both training and providing pre- and postgenetic education and counselling would be valuable in facilitating the mainstreaming of genetics in clinical care.

The purpose of reviews of this type is to determine whether there is sufficient evidence to inform the development of clinical recommendations. The current level of evidence to support genetic testing for melanoma, based on the subsequent effect on individuals' health behaviours, remains limited. The relationships between knowledge of risk, intention and motivation, and behaviour change are complex and require careful consideration of study design. For this cohort, personal experience including family history, education and access to services could present as potential confounders. The inclusion of variable study methods comprising quantitative, qualitative and hypothetical scenarios can hinder the synthesis of study outcomes, limiting inferences. The inclusion of all study types can equally be viewed as a strength of this review, as the aim was to capture all available data in this emerging field, to identify strengths and weaknesses of current evidence and where to focus future research. Grey literature and conference abstracts were not included in the literature search, therefore there is a risk of publication bias. While the quality of most studies reviewed was moderate to high, the level of evidence produced is weak according to "Levels of Evidence" guidelines, due to the lack of randomised controlled trials and reliance on cohort study design [53]. A sufficiently powered, "gold standard" randomised controlled trial is unlikely to be achieved, as the low incidence of mutation carriers and ethical issues of controlled randomisation are constraining. A further limitation on generalisability must be acknowledged, due to a homogeneous study population with higher than average household income and level of education, and mostly white Caucasian skin type. Furthermore, it is noted that the majority of the studies included in this review were conducted in 1 country, the USA, in which a large portion of participants were recruited from 2 extended CDKN2A-positive family kindreds in Utah. Further studies of different populations from diverse backgrounds would improve the generalisability and strengthen the evidence [54]. In many studies, a lack of follow-up of prevention outcomes, and small sample sizes, has inhibited meaningful conclusions on whether improvements in behaviour are sustained. Previous research has shown that adherence to skin surveillance does decrease over time. In high-risk individuals, it is not until year 4 or 5 that the rates of adherence decrease significantly [55]. Considering the cost of genetic testing and the increasing public demand for such services, highquality research in this area is important and necessary.

\section{Conclusion}

The evidence reviewed suggests that genetic testing for melanoma has a modestly positive impact on both primary and secondary preventive behaviour in high-risk individuals. Furthermore, improvements are observed regardless of mutation carrier status, although greater adherence is found in carriers. Although more robust trials, with long-term outcome follow-ups, are needed to inform the development of new clinical recommendations, the findings to date suggest a positive impact of genetic testing for melanoma on preventative behaviours. 


\section{Key Message}

Evidence suggests that genetic testing for familial melanoma can improve primary and secondary preventative behaviours.

\section{Statement of Ethics}

This is a literature review which did not involve human subject recruitment. Thus, ethical approval was not required.

\section{Conflict of Interest Statement}

H.P.S. is a shareholder of MoleMap NZ Ltd. and e-derm consult $\mathrm{GmbH}$, and undertakes regular teledermatological reporting for both companies. H.P.S. is a medical consultant for Canfield Scientific Inc., Revenio Research Oy and also a medical advisor for First Derm.

\section{Funding Sources}

C.A.P. is funded by an Australian Government Research Training Programme Scholarship. A.M.M.-L. is funded via an NHMRC ECF APP1158111. H.P.S. holds an NHMRC MRFF Next Generation Clinical Researchers Program Practitioner Fellowship (APP1137127).

\section{Author Contributions}

C.A.P. performed database search, screening, eligibility and quality assessment. T.Y. independently screened $10 \%$ of articles and assessed quality of $25 \%$ of articles. C.A.P. prepared the first draft of the manuscript. All co-authors contributed to writing, provided intellectual input and approved the final version of the paper.

\section{References}

1 Tas F, Erturk K. Paradox in melanoma: more patients fewer publications. Melanoma Res. 2020, Online ahead of print.

2 Cust AE, Harland M, Makalic E, Schmidt D, Dowty JG, Aitken JF, et al. Melanoma risk for CDKN2A mutation carriers who are relatives of population-based case carriers in Australia and the UK. J Med Genet. 2011 Apr;48(4): 266-72.

3 Potrony M, Badenas C, Aguilera P, Puig-Butille JA, Carrera C, Malvehy J, et al. Update in genetic susceptibility in melanoma. Ann Transl Med. 2015 Sep;3(15):210.

4 Salerni G, Carrera C, Lovatto L, Puig-Butille JA, Badenas C, Plana E, et al. Benefits of total body photography and digital dermatoscopy ("two-step method of digital follow-up") in the early diagnosis of melanoma in patients at high risk for melanoma. J Am Acad Dermatol. 2012 Jul;67(1):e17-27.

5 Nahar VK, Ford MA, Jacks SK, Thielen SP, Johnson AK, Brodell RT, et al. Sun-related behaviors among individuals previously diagnosed with non-melanoma skin cancer. Indian J Dermatol Venereol Leprol. 2015 NovDec;81(6):568-75.

6 Azzarello LM, Dessureault S, Jacobsen PB. Sunprotective behavior among individuals with a family history of melanoma. Cancer Epidemiol Biomarkers Prev. 2006 Jan;15(1):142-5.

7 Bertolin M, Cercatto MC, Requena C, SerraGuillen C, Llombart B, Sanmartin O, et al. Awareness, Attitude, and Adherence to Preventive Measures in Patients at High Risk of Melanoma. A Cross-Sectional Study on $185 \mathrm{~Pa}$ tients. J Cancer Educ. 2015 Sep;30(3):552-66.

8 Bishop JA, Taylor T, Potts HW, Elliott F, Pinney E, Barrett JH, et al. Sun-protective behaviors in families at increased risk of melanoma. J Invest Dermatol. 2007 Jun;127(6):1343-50.
9 Watts CG, Dieng M, Morton RL, Mann GJ, Menzies SW, Cust AE. Clinical practice guidelines for identification, screening and follow-up of individuals at high risk of primary cutaneous melanoma: a systematic review. Br J Dermatol. 2015 Jan;172(1):33-47.

10 Frost CJ, Andrulis IL, Buys SS, Hopper JL, John EM, Terry MB, et al. Assessing patient readiness for personalized genomic medicine. J Community Genet. 2019 Jan;10(1):109-20.

11 Wright MF, Lewis KL, Fisher TC, Hooker GW, Emanuel TE, Biesecker LG, et al. Preferences for results delivery from exome sequencing/genome sequencing. Genet Med. 2014 Jun;16(6):442-7.

12 Young C, Argáez C. CADTH Rapid Response Reports. Rapid Genome-wide Testing: A Review of Clinical Utility, Cost-Effectiveness, and Guidelines. Ottawa (ON): Canadian Agency for Drugs and Technologies in Health Copyright ${ }^{\circ} 2019$ Canadian Agency for Drugs and Technologies in Health.; 2019.

13 Botkin JR, Teutsch SM, Kaye CI, Hayes M, Haddow JE, Bradley LA, et al.; EGAPP Working Group. Outcomes of interest in evidencebased evaluations of genetic tests. Genet Med. 2010 Apr;12(4):228-35.

14 Fennell AP, Hunter MF, Corboy GP. The changing face of clinical genetics service delivery in the era of genomics: a framework for monitoring service delivery and data from a comprehensive metropolitan general genetics service. Genet Med. 2020 Jan;22(1):210-8.

15 Liberati A, Altman DG, Tetzlaff J, Mulrow C, Gøtzsche PC, Ioannidis JP, et al. The PRISMA statement for reporting systematic reviews and meta-analyses of studies that evaluate healthcare interventions: explanation and elaboration. BMJ. 2009 Jul;339:b2700.
16 Kmet LM, Lee RC. Standard Quality Assessment Criteria for Evaluating Primary Research Papers from a Variety of FieldsAHFMRHTA Initiative20040213. HTA Initiative. 2004;2.

17 Campbell M, Katikireddi SV, Sowden A, McKenzie JE, Thomson H. Improving Conduct and Reporting of Narrative Synthesis of Quantitative Data (ICONS-Quant): protocol for a mixed methods study to develop a reporting guideline. BMJ. 2018;8(2):e020064.

18 Hay JL, Gordon M, Li Y. Family risk discussions after feedback on genetic risk of melanoma. JAMA Dermatol. 2015 Mar;151(3): 342-3.

19 Taber JM, Aspinwall LG, Leaf SL, Kohlmann W, Leachman SA. Partner involvement in conduct of skin self-examinations remains low following CDKN2A/p16 genetic test reporting and counseling. J Am Acad Dermatol. 2013 Nov;69(5):842-4.

$20 \mathrm{McHugh}$ ML. Interrater reliability: the kappa statistic. Biochem Med (Zagreb). 2012;22(3): 276-82.

21 Kasparian NA, Meiser B, Butow PN, Job RF, Mann GJ. Better the devil you know? High-risk individuals' anticipated psychological responses to genetic testing for melanoma susceptibility. J Genet Couns. 2006 Dec;15(6):433-47.

22 Christensen KD, Roberts JS, Shalowitz DI, Everett JN, Kim SY, Raskin L, et al. Disclosing individual CDKN2A research results to melanoma survivors: interest, impact, and demands on researchers. Cancer Epidemiol Biomarkers Prev. 2011 Mar;20(3):522-9.

23 Leof ER, Zhu X, Rabe KG, McCormick JB, Petersen GM, Radecki Breitkopf C. Pancreatic cancer and melanoma related perceptions and behaviors following disclosure of CDK$\mathrm{N} 2 \mathrm{~A}$ variant status as a research result. Genet Med. 2019 Nov;21(11):2468-77. 
$24 \mathrm{Wu}$ YP, Aspinwall LG, Michaelis TC, Stump T, Kohlmann WG, Leachman SA. Discussion of photoprotection, screening, and risk behaviors with children and grandchildren after melanoma genetic testing. J Community Genet. 2016 Jan;7(1):21-31.

25 Wu YP, Aspinwall LG, Parsons B, Stump TK, Nottingham K, Kohlmann W, et al. Parent and child perspectives on family interactions related to melanoma risk and prevention after CDKN2A/p16 testing of minor children. J Community Genet. 2020 Jul;11(3):321-9.

26 Bergenmar M, Hansson J, Brandberg Y. Family members' perceptions of genetic testing for malignant melanoma-a prospective interview study. Eur J Oncol Nurs. 2009 Apr; 13(2):74-80.

27 Kasparian NA, Meiser B, Butow PN, Simpson JM, Mann GJ. Genetic testing for melanoma risk: a prospective cohort study of uptake and outcomes among Australian families. Genet Med. 2009 Apr;11(4):265-78.

28 Glanz K, Volpicelli K, Kanetsky PA, Ming ME, Schuchter LM, Jepson C, et al. Melanoma genetic testing, counseling, and adherence to skin cancer prevention and detection behaviors. Cancer Epidemiol Biomarkers Prev. 2013 Apr;22(4):607-14.

29 Aspinwall LG, Leaf SL, Kohlmann W, Dola ER, Leachman SA. Patterns of photoprotection following CDKN2A/p16 genetic test reporting and counseling. J Am Acad Dermatol. 2009 May;60(5):745-57.

30 Aspinwall LG, Taber JM, Kohlmann W, Leaf SL, Leachman SA. Unaffected family members report improvements in daily routine sun protection 2 years following melanoma genetic testing. Genet Med. 2014 Nov;16(11): 846-53.

31 Stump TK, Aspinwall LG, Drummond DM, Taber JM, Kohlmann W, Champine M, et al. CDKN2A testing and genetic counseling promote reductions in objectively measured sun exposure one year later. Genet Med. 2020 Jan; 22(1):26-34.

32 Aspinwall LG, Stump TK, Taber JM, Drummond DM, Kohlmann W, Champine M, et al. Genetic test reporting of CDKN2A provides informational and motivational benefits for managing melanoma risk. Transl Behav Med. 2018 Jan;8(1):29-43.

33 Stump TK, Aspinwall LG, Kohlmann W, Champine M, Hauglid J, Wu YP, et al. Genetic Test Reporting and Counseling for Melanoma Risk in Minors May Improve Sun Protection Without Inducing Distress. J Genet Couns. 2018 Aug;27(4):955-67.
34 Aspinwall LG, Leaf SL, Dola ER, Kohlmann W, Leachman SA. CDKN2A/p16 genetic test reporting improves early detection intentions and practices in high-risk melanoma families. Cancer Epidemiol Biomarkers Prev. 2008 Jun; 17(6):1510-9.

35 Aspinwall LG, Taber JM, Leaf SL, Kohlmann $\mathrm{W}$, Leachman SA. Melanoma genetic counseling and test reporting improve screening adherence among unaffected carriers 2 years later. Cancer Epidemiol Biomarkers Prev. 2013 Oct;22(10):1687-97.

36 Levin T, Mæhle L. Uptake of genetic counseling, genetic testing and surveillance in hereditary malignant melanoma (CDKN2A) in Norway. Fam Cancer. 2017 Apr;16(2):25765.

37 Hanson CL, Crandall A, Barnes MD, Magnusson B, Novilla ML, King J. Family-Focused Public Health: Supporting Homes and Families in Policy and Practice. Front Public Health. 2019 Mar;7:59.

38 Barnes MD, Hanson CL, Novilla LB, Magnusson BM, Crandall AC, Bradford G. FamilyCentered Health Promotion: Perspectives for Engaging Families and Achieving Better Health Outcomes. Inquiry. 2020 Jan-Dec;57: 46958020923537

39 Hansson J. Familial cutaneous melanoma. Adv Exp Med Biol. 2010;685:134-45.

40 Wei EX, Li X, Nan H. Having a first-degree relative with melanoma increases lifetime risk of melanoma, squamous cell carcinoma, and basal cell carcinoma. J Am Acad Dermatol. 2019 Aug;81(2):489-99.

41 Ransohoff KJ, Jaju PD, Tang JY, Carbone M, Leachman S, Sarin KY. Familial skin cancer syndromes: increased melanoma risk. J Am Acad Dermatol. 2016 Mar;74(3):423-34.

42 Schwartz MD, Isaacs C, Graves KD, Poggi E, Peshkin BN, Gell C, et al. Long-term outcomes of BRCA1/BRCA2 testing: risk reduction and surveillance. Cancer. 2012 Jan; 118(2):510-7.

43 Petelin L, Hossack L, Shanahan M, Mitchell G, Liew D, James PA, et al. Cost-effectiveness of long-term clinical management of BRCA pathogenic variant carriers. Genet Med. 2020 May;22(5):831-9.

44 Heshka JT, Palleschi C, Howley H, Wilson B, Wells PS. A systematic review of perceived risks, psychological and behavioral impacts of genetic testing. Genet Med. 2008 Jan;10(1) 19-32.
45 Frieser MJ, Wilson S, Vrieze S. Behavioral impact of return of genetic test results for complex disease: systematic review and metaanalysis. Health Psychol. 2018 Dec;37(12): 1134-44.

46 Hollands GJ, French DP, Griffin SJ, Prevost AT, Sutton S, King S, et al. The impact of communicating genetic risks of disease on riskreducing health behaviour: systematic review with meta-analysis. BMJ. 2016 Mar;352:11 102.

47 Smerecnik C, Grispen JE, Quaak M. Effectiveness of testing for genetic susceptibility to smoking-related diseases on smoking cessation outcomes: a systematic review and metaanalysis. Tob Control. 2012 May;21(3):34754.

48 Marteau TM, French DP, Griffin SJ, Prevost AT, Sutton S, Watkinson C, et al. Effects of communicating DNA-based disease risk estimates on risk-reducing behaviours. Cochrane Database Syst Rev. 2010 Oct;(10):CD007275.

49 Yanes T, Willis AM, Meiser B, Tucker KM, Best M. Psychosocial and behavioral outcomes of genomic testing in cancer: a systematic review. Eur J Hum Genet. 2019 Jan;27(1): 28-35.

50 McCuaig JM, Armel SR, Care M, Volenik A, Kim RH, Metcalfe KA. Next-Generation Service Delivery: A Scoping Review of Patient Outcomes Associated with Alternative Models of Genetic Counseling and Genetic Testing for Hereditary Cancer. Cancers (Basel). 2018 Nov; 10(11):E435.

51 O'Shea R, Rankin NM, Kentwell M, Gleeson M, Salmon L, Tucker KM, et al. How can Australia integrate routine genetic sequencing in oncology: a qualitative study through an implementation science lens. Genet Med. 2020 Sep;22(9):1507-16.

52 Fuertes JN, Mislowack A, Bennett J, Paul L, Gilbert TC, Fontan G, et al. The physicianpatient working alliance. Patient Educ Couns. 2007 Apr;66(1):29-36.

53 Burns PB, Rohrich RJ, Chung KC. The levels of evidence and their role in evidence-based medicine. Plast Reconstr Surg. 2011 Jul; 128(1):305-10.

54 Rothwell PM. External validity of randomised controlled trials: "to whom do the results of this trial apply?”. Lancet. 2005 Jan;365(9453): 82-93.

55 Reserva J, Janeczek M, Joyce C, Goslawski A, Hong H, Yuan FN, et al. A Retrospective Analysis of Surveillance Adherence of $\mathrm{Pa}$ tients after Treatment of Primary Cutaneous Melanoma. J Clin Aesthet Dermatol. 2017 Dec;10(12):44-8 